\title{
Tunnel personnel positioning method based on TOA and modified location-fingerprint positioning
}

\author{
Sun Jiping ${ }^{\mathrm{a}}$, Li Chenxin ${ }^{\mathrm{a}, \mathrm{b}, *}$
}

${ }^{a}$ School of Mechanical Electronic \& Information Engineering, China University of Mining \& Technology, Beijing 100083, China

${ }^{b}$ Branch of Mine Safety Equipment Technology, China Coal Research Institute, Beijing 100013, China

Abstract: To position personnel in mines, the study discussed in this paper built on the tunnel personnel positioning method on the basis of both TOA and location-finger print (LFP) positioning. Given non-line of sight (NLOS) time delay in signal transmission caused by facilities and equipment shielding in tunnels and TOA measurement errors in both LFP database data and real-time data, this paper puts forth a database data de-noising algorithm based on distance threshold limitation and modified mean filtering (MMF), as well as a real-time data suppression algorithm based on speed threshold limitation and MMF. On this basis, a nearest neighboring data matching algorithm based on historical location and the speed threshold limitation is used to estimate personnel location and realize accurate personnel positioning. The results from both simulation and the experiment suggest that: compared with the basic LFP positioning method and the method that only suppresses real-time data error, the tunnel personnel positioning methods based on TOA and modified LFP positioning permits effectively eliminating error in TOA measurement, making the measured data close to the true positional data, and dropping the positioning error: the maximal positioning error in measurements from experiment drops by 9 and $3 \mathrm{~m}$, respectively, and the positioning accuracy of $3 \mathrm{~m}$ is achievable in the condition used in the experiment.

Keywords: Tunnel; Positioning; TOA; Location-finger print (LFP); Modified mean filtering (MMF); Distance threshold; Speed threshold

\section{Introduction}

Mine accurate positioning, a vital technologic means to ensure personnel safety in mine production, is one of key technologies in researches concerning "six systems” for mine safety protection [1-4]. The location-finger print (LFP) positioning method, an accurate positioning method and suitable for studying indoor accurate positioning, is mainly achieved through the ranging-based received signal strength indication (RSSI) and time of arrival (TOA) [5-9].

Some fundamental researches, mainly RSSI based, have been used on the LFP-positioning-based mine personnel accurate positioning method. The paper presented a WiFi-based mine positioning algorithm and proposed a RSSI-based LFP positioning method to estimate positions of individuals [10]. The paper put forth a RSSI-based kernel-function matching algorithm to carry out the mine LFP positioning [11]. The paper brought forth a modified mine LFP positioning matching algorithm based on RSSI and combining the K-nearest neighbor algorithm and the shortest historical path matching algorithm [12]. The paper presented the experiment on the mine LFP positioning algorithm on the basis of Wi-Fi and RSSI, and the result indicated that within the range of $25 \mathrm{~m}$, more accurate positioning could be attained [13].

There has so far been limited research on application of TOA-based technology in mine positioning, mainly because TOA technology needs high temporal synchronization among nodes and thus imposes high requirement on hardware $[11,12]$. This led to few papers on TOA-based tunnel LFP positioning methods. However, the paper came up with a mine TOA positioning method on the basis of Kalman filtering and fingerprint positioning, and discussed applying TOA-based LFP positioning in mines [14]. However, more parameters found in further research have to be determined if a Kalman filter is applied in mines, which may affect the final positioning result to some degree.

There are some shortcomings in researches on mine LFP positioning methods presented in papers. Because there are many factors affecting transmission of electro-magnetic waves (EMW) in tunnels, processing the real-time measurements alone cannot assure the method is reliable, and on the contrary, the error suppression of the real-time measurements and de-noising of data in the database are required synchronously [10-14].

By analyzing the impact of mine environment on RSSI and TOA technologies, the paper demonstrated that TOA technology is better-suited for positioning in tunnels and the LFP positioning method, which is feasible in the current condition of technology. By studying and improving TOA-based tunnel LFP positioning methods, considering the tunnel environment's effect on TOA ranging, the paper presented a real-time measurement error suppression method and a database data de-noising algorithm both of which require fewer parameters, to position personnel in tunnels at high accuracy.

\section{Comparison between TOA and RSSI \\ 2.1 Resolution}

RSSI is calculated with the formula below using the received strength of wireless signals:

$$
P(d)=-10 n \log \left(\frac{d}{d_{0}}\right)+P\left(d_{0}\right)+A
$$

where $P(d)$ is the received strength of wireless signal; $P\left(d_{0}\right)$ the strength reference at $d_{0}$; and $A$ the additional loss due to environment, i.e., due to tunnel. Ideally, the free-space transmission model may be applied in analysis and the relation between RSSI value and the result from ranging is presented as shown in Fig. 1.

Received 15 August 2015; revised 09 December 2015; accepted 10 January 2016

*Corresponding author. Tel.: +86 15210198505

E-mail address: cumtblichenxin@126.com 


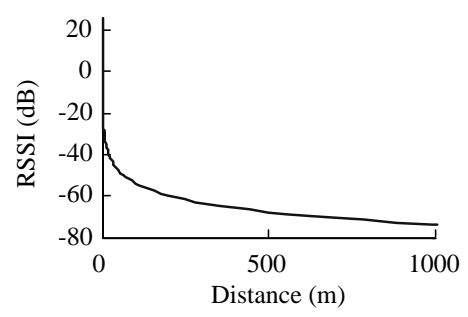

Fig. 1. RSSI curve in the ideal condition

It is evident from Fig. 1 that in the ideal condition, the RSSI-based ranging resolution decreases with increase in the distance between the receiver and the transmitter, and the accuracy is high when the distance is small.

TOA is calculated with the formula below using the travel time of wireless signals:

$$
d=c \times t_{T O A}
$$

where $d$ is the traveling distance, i.e., the result from ranging; $c$ the light's speed; and $t_{T O A}$ the travel time of wireless signals. The relation between TOA and the result from ranging in the ideal condition is shown in Fig. 2.

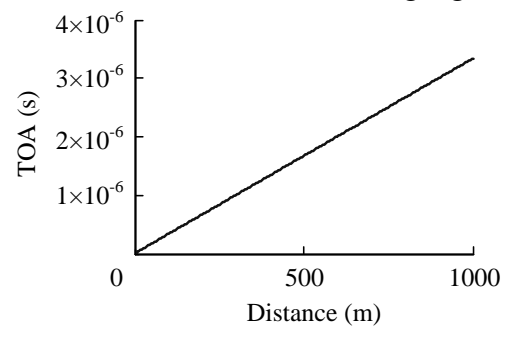

Fig. 2. TOA curve in the ideal condition

The TOA-based ranging result is linearly corresponding to TOA at a constant rate of change and the obtained ranging accuracy, which correlates with the temporal resolution of TOA, is expressed as follows [15]:

$$
P_{\text {Range }}=\tau \times c
$$

where $P_{\text {Range }}$ is the ranging accuracy; and $\tau$ the temporal resolution.

\subsection{RSSI in mine environment}

There are many factors leading to losses in EMW transmission in tunnels, including conventional free space losses, EMW tunnel waveguide losses, the tunnel wall roughness and inclination losses, metallic facilities shielding losses. With all factors considered, RSSI distribution of $2.4 \mathrm{GHz}$ band EMW in tunnel is shown in Fig. 3. The analysis method is the mode superposition of EMW transmission in tunnel $[16,17]$.

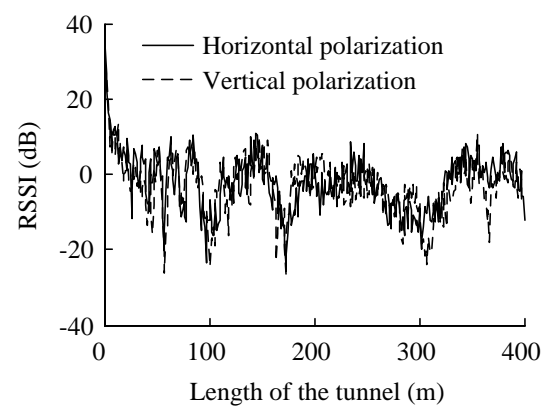

Fig. 3. RSSI curve in mine tunnels

From the Fig. 3, it is obvious that RSSI resolution drops with increasing wireless signal influenced by tunnel losses. For $2.4 \mathrm{GHz}$ signal polarized whether horizontally or vertically, ranging cannot be carried out with losses to a certain degree. The curve with wide fluctuation range results in the same received strength corresponding to multiple ranging distances. For the case shown in the Fig. 3, it is very difficult to distinguish the specific locations of the positioning targets in the area beyond $30 \mathrm{~m}$.

\subsection{TOA in mine environment}

Cui et al. concluded that TOA technology requires high temporal synchronization among nodes. However, GPS is capable of high temporal synchronization in ground TOA positioning but cannot be applied in mines $[11,12]$. For this reason, few researches have been conducted concerning TOA application in mine positioning.

In the tunnel environment of mines, errors in TOA technology include the synchronization time-delay error inherent to the technology, the timing error and NLOS time-delay error attributive to shielding of facilities and equipment inside the tunnel. 


$$
t_{\mathrm{TOA}}^{\prime}=t_{\mathrm{TOA}}+t_{\mathrm{SYNC}}+t_{\mathrm{e}}+t_{\mathrm{NLOS}}
$$

where $t_{\mathrm{TOA}}$ is the actual travel time of signals from the transmitting end to the receiving end; $t_{\mathrm{SYNC}}$ the time-synchronization error, i.e., the synchronization time-delay between the devices at the transmitting end and at the receiving end; $t_{\mathrm{e}}$ the timing error, dependent on the biasing of frequency of the crystal oscillator in the timer; and $t_{\text {NLOS }}$ the NLOS time-delay. All of the three factors act independently of each other.

The two-way ranging method can get rid of $t_{\mathrm{SYNC}}$, and the symmetric double-sided two-way ranging (SDS-TWR) TOA method can eradicate $t_{\mathrm{SYNC}}$ and mitigate effect of $t_{\mathrm{e}}$ on positioning $[14,18]$.

Therefore, with the currently available technologies, the key to application of TOA technology in tunnels is to restrain the NLOS time delay. The NLOS time delay is present in EMW traveling due to shielding of facilities and equipment in tunnels, including the fixed ones such as signboards, overhead lines and ventilation devices, and the mobile ones that appear in tunnels any time such as vehicles. Aiming at the effect of the two kinds of NLOS time delay, TOA of the tunnel EMW travel is simulated, where the reference NLOS time-delay model developed in the paper is used for the fixed NLOS time-delay $[14,19]$.

$$
\tau_{\mathrm{NLOS}}=T_{\tau} d^{\varepsilon} \xi
$$

where $T_{\tau}$ is the median of NLOS time delay, which is set as 0.75 us; $\varepsilon$ an exponent between 0.5 and 1 , assumed to be 0.75 ; $\xi$ the random variable subject to the $\operatorname{logarithmic}$ distribution, i.e. $10 \lg \xi$, a Gaussian random variable of which the mean is 0 and the standard deviation (SD) $\delta_{\xi}$ is $4-6 \mathrm{~dB}$; and $d$ the physical distance. In simulation, the values of other parameters include the error variance of measured distance represented by Gaussian white noise is 1, and the range of error due to sudden NLOS time-delay attributive to random factors is from 10 to $20 \mathrm{~m}$.

TOA simulation for EMW travel in tunnel is shown in Fig. 4.

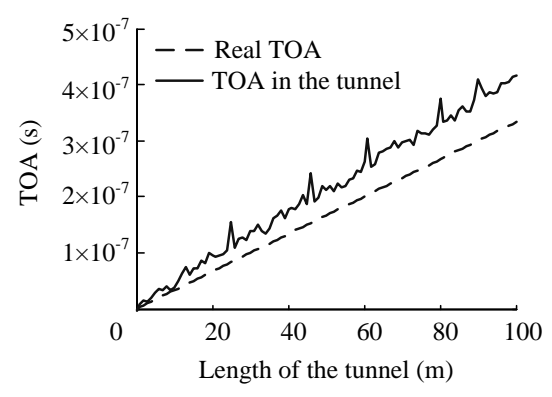

Fig. 4. TOA curve in mine tunnels

Though the curve undulates, TOA increases largely with increase in tunnel length and does not occur significantly that one single TOA value corresponds to a few distances (Fig. 4).

\subsection{Direction of study on tunnel LFP positioning}

In terms of both theoretical resolution and tunnel's effects, RSSI technology can deliver high ranging accuracy only at a close distance between the receiver and the transmitter while the ranging accuracy of TOA technology is not affected even at longer distance; in comparison, the fact that measured value may correspond to a few of distances occur for RSSI within a rather large range due to factors existed in tunnels while it is not the case for TOA technology. Therefore, where hardware permits, TOA technology is better suited for accurate positioning in mine tunnel environment.

On the other hand, unlike conventional positioning methods, LFP positioning is a mode-matching method, comprised of two processes: the processes to set up an offline database and to match the real-time measurements and the data in database $[20,21]$. For the two processes, the higher the distinction degree of the fingerprint data is, the higher the positioning accuracy becomes. Therefore, according to previous analysis, TOA technology is better suited for LFP positioning than RSSI technology.

The detailed process of LFP positioning in a specific tunnel is shown in Fig. 5; and for this, an offline database had to be developed first, as shown in the right half of Fig. 5. 


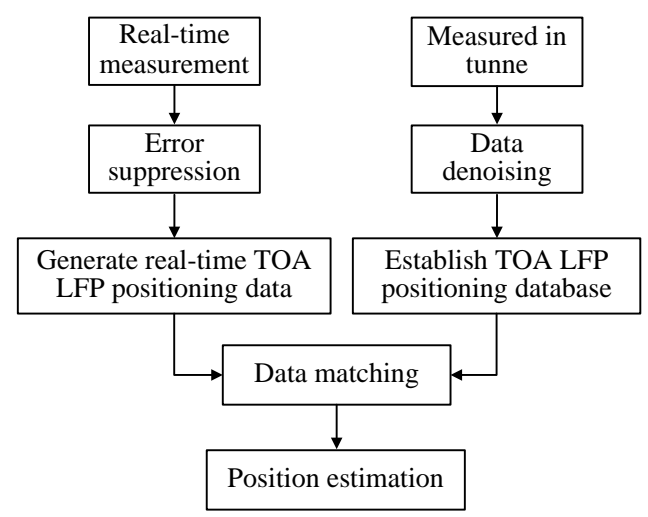

Fig. 5. Block diagram of TOA-based tunnel LFP positioning method

It is known from previous analysis that whether during real-time measurement or during development of tunnel LFP positioning database, shielding of mobile vehicles and other facilities/equipment may possibly take place, and therefore one has to combine de-noising of tunnel LFP positioning database and error suppression of real-time measurements in the investigation to obtain desired positioning accuracy.

Therefore, there are three key aspects in TOA-based tunnel LFP positioning method: de-noise data in LFP positioning database, suppress error in measured data in real time, and match the real-time data and database data. Some researchers have come up with solutions to the above-mentioned three aspects, respectively. For example, Liu et al. mainly focused on the last two aspects while Sun et al. argued that measurement for database should be made when such random factors as mobile vehicles are not present [10-14]. No achievement has been made from researches on any effective solutions as a whole to technical bottles present in TOA-based tunnel LFP positioning method in the physical condition of coal mines.

In accordance with characteristics in TOA measurement in tunnels, this paper presents a database data de-noising algorithm based on distance threshold limitation and modified mean filtering (MMF), and a real-time data error suppression algorithm based on speed threshold limitation and MMF.

\section{TOA-based modified tunnel LFP positioning method}

\subsection{SDS-TWR-based TOA measurement}

The philosophy of SDS-TWR (symmetric double-sided two way ranging) is illustrated in Fig. 6 [14].

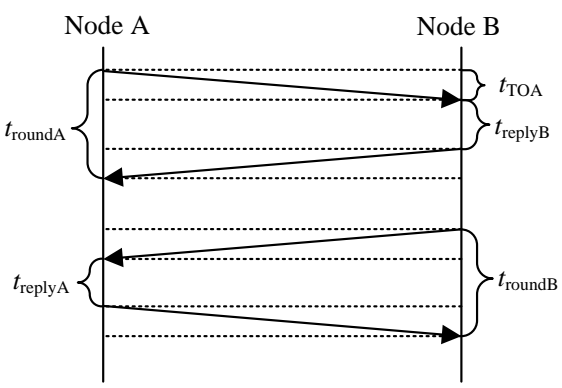

Fig. 6. Sketch of SDS-TWR method

It includes measuring TWR with double-sided signal travel time twice: for the first TWR, node A is the transmitting end and node B is the receiving end; for the second TWR, node B is the transmitting end and node A is the receiving end.

The time measured is

$$
t_{\mathrm{TOA}}=\frac{t_{\text {roundA }}-t_{\text {replyB }}+t_{\text {roundB }}-t_{\text {replyA }}}{4}
$$

The result from SDS-TWR-based TOA Measurement is

$$
t_{\mathrm{TOA}}^{\prime}=t_{\mathrm{TOA}}+t_{\mathrm{NLOS}}
$$

Therefore, TOA measurement with SDS-TWR can effectively remove effects of both synchronization time delay and timing error, and what one has to do is just dealing with NLOS time-delay error. For this reason, the issue of high temporal synchronization among nodes required for TOA technology is not a question any more.

The system structure for the positioning method is provided in Fig. 7. 


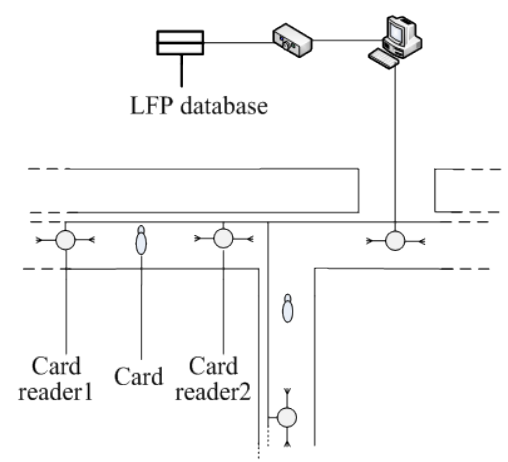

Fig. 7. System structure for the positioning

The one-dimension double-station position is used as the positioning method; and in the text below, the double stations are marked as card reader 1 and card reader 2 .

\subsection{Database data de-noising algorithm}

Because of effect of NLOS time delay in tunnel on TOA measurements shown in Fig. 4, those values that change greatly due to sudden appearance of facilities and equipment such as moving vehicles shall be removed as noise; on the other hand, because measured TOA values rise with increase in distance, the measured value distribution curve shall smooth in favor of data matching.

Because people walking in tunnels are unlikely to change their location for a longer distance all of a sudden, a sudden change in a distance value measured with TOA within a larger range may be considered as the result from MLOS time delay in EMW transmission due to equipment such as moving vehicles. Therefore, the distance threshold is expressed in the formula below:

$$
d_{T s}=(5+\Delta d) m
$$

where $\Delta \boldsymbol{d}$ is the interval of sampled distance for the LFP positioning database and the distance threshold is the value plus the error range of $5 \mathrm{~m}$.

On the condition that the distance threshold is limited, the author put forth the modified mean filtering (MMF) to de-noise data in the database and, any data, if the absolute value of difference between it and neighboring data is beyond the threshold, will be discarded and replaced with the mean of neighboring data so as to de-noise LFP positioning database because measured TOA values increase with increase in distance.

The first step for MMF of measured data in database is to determine, discard and replace the data beyond threshold:

$$
\begin{aligned}
& d_{1}^{*}(x)=\left\{\begin{array}{l}
d_{1}^{\prime}(x)\left|d_{1}^{\prime}(x)-d_{1}^{*}(x-\Delta d)\right|<d_{t s} \\
\frac{1}{2 l}\left(\sum_{i=-l}^{-1} d_{1}^{*}(x+i \Delta d)+\sum_{j=1}^{l} d_{1}^{\prime}(t+j \Delta d)\right),\left|d_{1}^{\prime}(x)-d_{1}^{*}(x-\Delta d)\right| \geq d_{t s}
\end{array}\right. \\
& d_{2}^{*}(x)=\left\{\begin{array}{l}
d_{2}^{\prime}(x), d_{2}^{\prime}(x)-d_{2}^{*}(x-\Delta d) \mid<d_{t s} \\
\frac{1}{2 l}\left(\sum_{i=-l}^{-1} d_{2}^{*}(x+i \Delta d)+\sum_{j=1}^{l} \dot{d_{2}}(t+j \Delta d)\right), \mid d_{2}^{\prime}(x)-d_{2}^{*}(x-\Delta d) \geq d_{t s}
\end{array}\right.
\end{aligned}
$$

where $d^{\prime}(x)$ is the measure data of developed LFP positioning database; $x$ the distance between the measure point and the card reader; $d^{*}(x)$ the data processed from the first step of MMF; and $l$ the radius of the neighborhood.

The second step for MMF algorithm is to smooth data;

$$
\begin{aligned}
& d_{1}(x)=\frac{1}{2 l+1}\left(\sum_{i=-l}^{l} d_{1}^{*}(x+i \Lambda d)\right) \\
& d_{2}(x)=\frac{1}{2 l+1}\left(\sum_{i=-l}^{l} d_{2}^{*}(x+i \Lambda d)\right)
\end{aligned}
$$

where $d(x)$ is the data processed from MMF and stored in the LFP positioning database.

The data format in the fingerprint database is

$$
F(x)=\left((1,2),\left(d_{1}(x), d_{2}(x)\right),\left(d_{1}^{r}(x), d_{2}^{r}(x)\right)\right)
$$

where $(1,2)$ is the ID information of the card reader; $\left(d_{1}(x), d_{2}(x)\right)$ the pre-stored measured value processed 
from MMF at the point; and $\left(d_{1}^{r}(x), d_{2}^{r}(x)\right)$ the true value at the point.

\subsection{Real-time data error suppression algorithm}

Similarly, large undulation exists in the real-time data due to sudden appearance of facilities and equipment such as moving vehicles and thus shall be suppressed as error. The speed-threshold limitation is used in MMF to suppress errors in real-time data. The speed threshold is:

$$
v_{T s}=\left(2+\frac{5}{T}\right) m / s
$$

where $T$ is the positioning patrol cycle and the speed threshold is derived by the moving distance in a positioning patrol cycle adding an error range of $5 \mathrm{~m}$.

The first step for MMF based error suppression of real-time data is

$$
\begin{aligned}
& d_{1}^{*}(t)=\left\{\begin{array}{l}
d_{1}^{\prime}(t),\left|d_{1}^{\prime}(t)-d_{1}^{*}(t-T)\right|<T \times v_{T s} \\
\frac{1}{2 l}\left(\sum_{i=-l}^{-1} d_{1}^{*}(t+i T)+\sum_{j=1}^{l} d_{1}^{\prime}(t+j T)\right),\left|d_{1}^{\prime}(t)-d_{1}^{*}(t-T)\right| \geq T \times v_{T s}
\end{array}\right. \\
& d_{2}^{*}(t)=\left\{\begin{array}{l}
d_{2}^{\prime}(t),\left|d_{2}^{\prime}(t)-d_{2}^{*}(t-T)\right|<T \times v_{T s} \\
\frac{1}{2 l}\left(\sum_{i=-l}^{-1} d_{2}^{*}(t+i T)+\sum_{j=1}^{l} d_{2}^{\prime}(t+j T)\right),\left|d_{2}^{\prime}(t)-d_{2}^{*}(t-T)\right| \geq T \times v_{T s}
\end{array}\right.
\end{aligned}
$$

where $d^{\prime}(t)$ is the data measured in real time by the card reader at the time $t$; and $d^{*}(t)$ the data processed from the first step of MMF.

The second step is to smooth the real-time data:

$$
\begin{aligned}
& d_{1}(t)=\frac{1}{2 l+1}\left(\sum_{i=-l}^{l} d_{1}^{*}(t+i T)\right) \\
& d_{2}(t)=\frac{1}{2 l+1}\left(\sum_{i=-l}^{l} d_{2}^{*}(t+i T)\right)
\end{aligned}
$$

where $d(t)$ is the measured real-time data after MMF.

The format of the real-time fingerprint data is

$$
F_{?}(t)=\left((1,2),\left(d_{1}(t), d_{2}(t)\right),\left(d_{1}^{?}(t), d_{2}^{?}(t)\right)\right)
$$

where $\left(d_{1}(t), d_{2}(t)\right)$ is the measured real-time data at the time $t$ after it is processed with MMF; and $\left(d_{1}^{?}(t), d_{2}^{?}(t)\right)$ the positional estimate at the point.

\subsection{Data matching algorithm}

Because sudden change in the location of walking people in tunnels is unlikely, matching the real-time data and the database data is based on the historical position and the speed threshold limitation: within the limited range of speed threshold, using Euclidean distance is to determine the nearest data and then estimate the position of personnel at the time $t$, i.e. the minimal Euclidean distance between a data in the database and the real-time data is obtained from calculation [22].

$$
L_{\min }\left(F(x), F_{?}(t)\right)=\left(\sqrt{\sum_{j=i}^{i+1}\left(d_{j}-d_{j}^{*}(t)\right)^{2}}\right)_{\text {min }}
$$

$\left(d_{1}^{r}(x), d_{2}^{r}(x)\right)$ contains that the fingerprint data of the database is the estimated position of personnel at the time $t$.

\section{Analysis of experimental results}

\subsection{Analysis of simulation}

In simulation, values of the fixed NLOS time delay and the sudden NLOS time delay are the same as the parameters used in TOA simulation for tunnel EMW transmission in Fig. 4. Additionally, the radius of the neighborhood is 2 during filtering.

The simulation analysis compares real-time data, the measured value of database data and filtering-processed data, including three parts. Fig. 8 shows the de-noising effect on database data on the basis of distance threshold limitation and MMF algorithm, as well as the error suppression effect on real-time data based on speed threshold limitation and MMF algorithm. In simulation experiment, calculation of real-time data with different processing methods and database data corresponding to the real locations reflects Euclidean distance distribution. The 
calculated Euclidean distances between the measured data and the database 'data of the real locations are significantly reduced by the LFP positioning method improved in this paper (Fig. 9). This is the key process reducing positioning error with LFP positioning method. The final positioning error distribution is shown in Fig. 10.

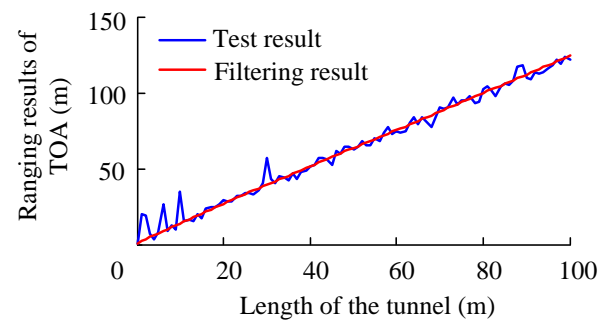

(a) Real-time data of card reader1

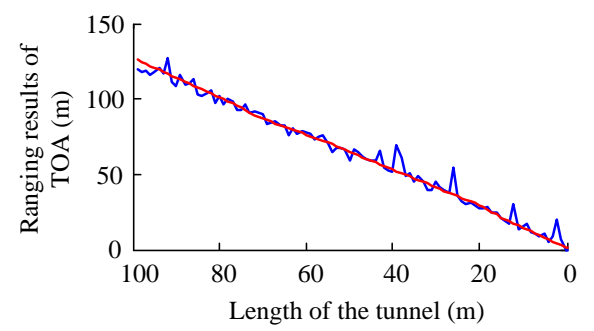

(c) Real-time data of card reader 2

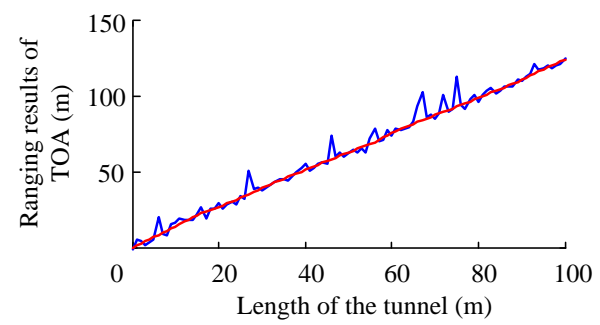

(b) Database data of card reader1

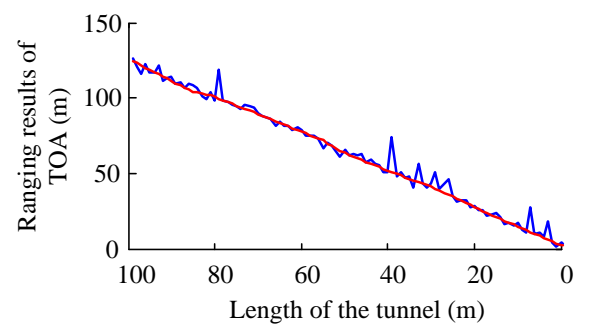

(d) Database data of card reader2

Fig. 8. TOA-based measured data and filtering-processed data

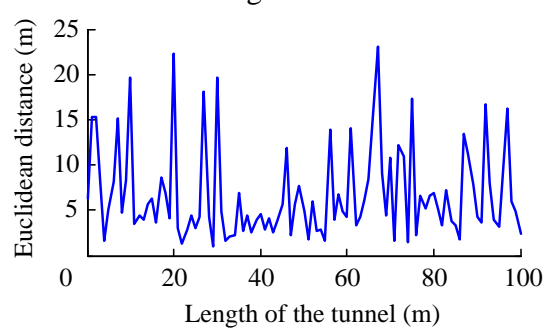

(a) Matching between two kinds of test data

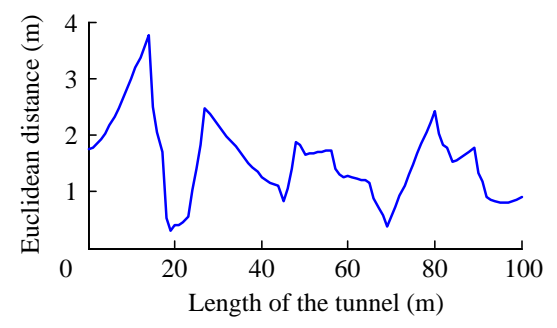

(c) Matching between two kinds of filtering data Comparison of Euclidean distance between the real

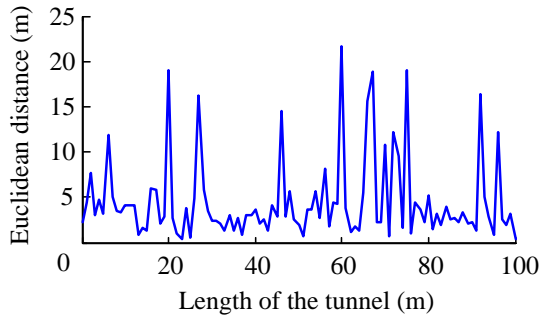

(b) Matching between real-time filtering and database test data

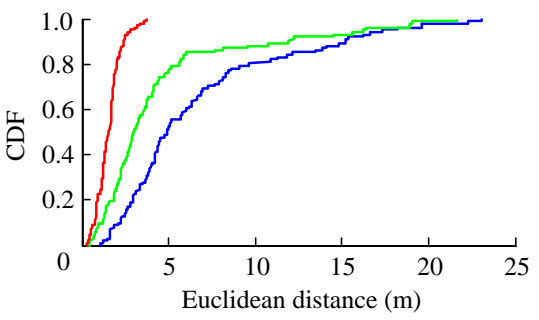

(d) CDF of euclidean distance of three methods time data and the data at the real positions using different

Fig. 9. Comparison of Euclidean distance between the real-time data and the data at the real positions using different processing methods

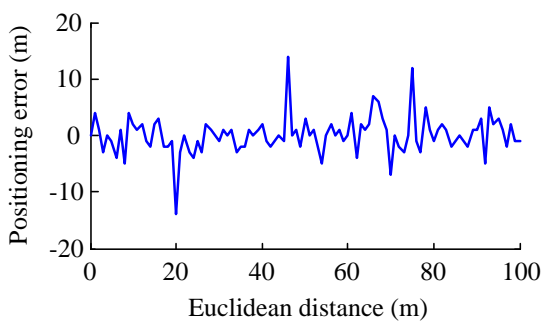

(a) Matching between two kinds of test data

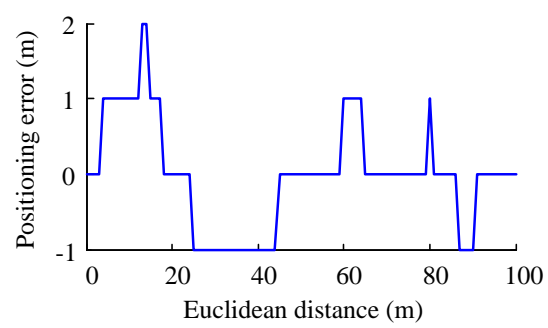

(c) Matching between two kinds of filtering

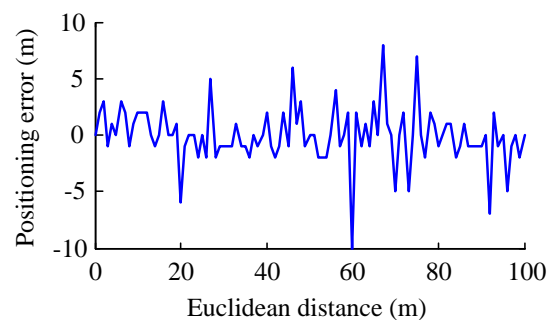

(b) Matching between real-time filtering and database test data

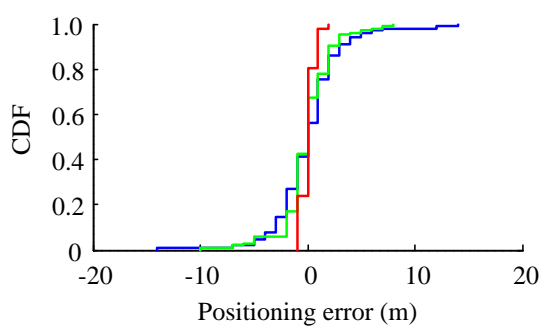

(d) CDF of positioning errors of three methods 
Fig. 10. Comparison of positioning error from different processing methods

From Fig. 8, among the real-time data and the database data filtered with the method presented in this paper, those with large deviation are effectively suppressed, so are the undulation.

It is observed from Fig. 9 that Euclidean distance between the measured values and the database data at real positions is calculated in three cases: obvious fluctuation in the values calculated from directly matching the real-time measured values and the measured values in database with the range from 0 to $23 \mathrm{~m}$ (blue CDF curve of Fig. 9d), suppressed fluctuation in the values calculated from matching the real-time measures values after MMF processing but with big calculated Euclidean distances at some points (green CDF curve of Fig. 9d). According to the method given in this paper, the calculated Euclidean distances are significantly reduced after MMF processing of both the real-time measured values and the measured values of the database data (red CDF curve of Fig. 9d). Consequently, this means that the method given in this paper is able to significantly reduce the calculated Euclidean distances between the real-time data and the database data of real locations so as to greatly increase accuracy in positional estimation.

The positioning errors of three simulation cases are shown in Fig. 10. Big positioning errors come from directly matching the real-time measured values and the measured values in the database, ranging from -14 to 13 m (blue CDF curve of Fig. 10d). Positioning errors are reduced when matching the real-time measures values after MMF processing and the measured values in database, ranging from -10 to $8 \mathrm{~m}$ (green CDF curve of Fig. 10d). For the final positioning results of the method given in this paper, the range of positioning errors is from -1 to $2 \mathrm{~m}$ (red CDF curve of Fig. 10d). Thus the method given in this paper can realize accurate personnel positioning in tunnels.

\subsection{Experiment and discussion}

The experiment was conducted inside a $40 \mathrm{~m}(L) \times 4 \mathrm{~m}(W) \times 3.5 \mathrm{~m}(H)$ tunnel at an exercise training base of a coal company wherein a number of equipment, metallic supports, overhead lines and hydraulic supports are provided. Card readers were installed $5 \mathrm{~m}$ from both ends. Both card readers and identification cards were equipped with TOA ranging module adopting SDS-TWR and using German Nanotron NA5TR1 as the core. TOA ranging at the designated points required in the database and the real-time TOA ranging for people walking at an even speed were conducted respectively. For ranging at the designated points, measurements were made every 1 $\mathrm{m}$ on the median line and for real-time ranging at even speed of walking, it was measured at the speed of $1 \mathrm{~m} / \mathrm{s}$ in real time every $1 \mathrm{~s}$. During the measurement, metallic equipment randomly travels in the tunnel. The experiment environment is as shown in Fig. 11.

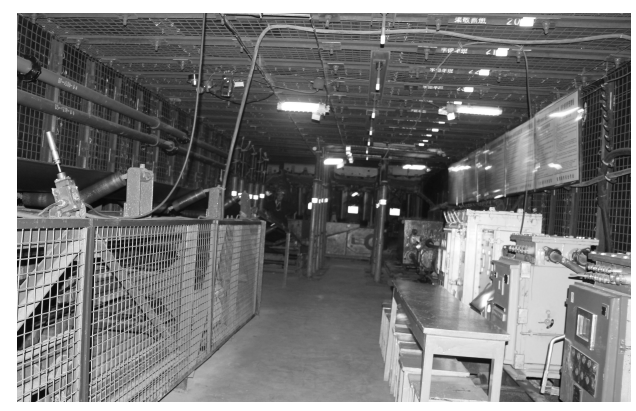

Fig. 11. Experiment site

The measured values are provided in Table 1.

Table 1 Measured data

\begin{tabular}{|c|c|c|c|c|c|c|c|}
\hline $\begin{array}{l}\text { Measurement } \\
\text { time (s) }\end{array}$ & $\begin{array}{l}\text { Measurement } \\
\text { data }(\mathrm{m})\end{array}$ & $\begin{array}{l}\text { Measurement } \\
\text { location (m) }\end{array}$ & $\begin{array}{l}\text { Database data } \\
\text { (m) }\end{array}$ & $\begin{array}{l}\text { Measurement } \\
\text { time (s) }\end{array}$ & $\begin{array}{l}\text { Measurement } \\
\text { data }(\mathrm{m})\end{array}$ & $\begin{array}{l}\text { Measurement } \\
\text { location (m) }\end{array}$ & $\begin{array}{l}\text { Database data } \\
(\mathrm{m})\end{array}$ \\
\hline 1 & $(0.67,40.95)$ & $(0,30)$ & $(1.45,41.15)$ & 17 & $(24.10,20.15)$ & $(16,14)$ & $(23.29,20.90)$ \\
\hline 2 & $(5.01,45.33)$ & (1.29) & $(2.24,39.21)$ & 18 & $(24.99,22.60)$ & $(17,13)$ & $(27.77,22.19)$ \\
\hline 3 & $(6.05,40.54)$ & $(2,28)$ & $(13.44,38.66)$ & 19 & $(26.80,21.08)$ & $(18,12)$ & $(28.73,16.74)$ \\
\hline 4 & $(20.17,36.42)$ & $(3,27)$ & $(9.35,37.56)$ & 20 & $(32.60,21.10)$ & $(19,11)$ & $(26.41,21.26)$ \\
\hline 5 & $(9.54,49.13)$ & $(4,26)$ & $(8.83,37.44)$ & 21 & $(28.73,15.58)$ & $(20,10)$ & $(29.23,18.70)$ \\
\hline 6 & $(12.20,34.20)$ & $(5,25)$ & $(10.16,34.94)$ & 22 & $(43.81,16.57)$ & $(21,9)$ & $(30.16,14.12)$ \\
\hline 7 & $(9.17,32.60)$ & $(6,24)$ & $(11.69,35.25)$ & 23 & $(33.07,27.89)$ & $(22,8)$ & $(29.93,15.06)$ \\
\hline 8 & $(11.12,34.58)$ & $(7,23)$ & $(11.86,35.84)$ & 24 & $(31.22,14.54)$ & $(23,7)$ & $(33.25,12.15)$ \\
\hline 9 & $(14.12,33.48)$ & $(8,22)$ & $(14.06,29.09)$ & 25 & $(36.76,9.28)$ & $(24,6)$ & $(33.75,10.39)$ \\
\hline 10 & $(16.63,31.20)$ & $(9,21)$ & $(13.69,31.19)$ & 26 & $(36.39,12.37)$ & $(25,5)$ & $(35.30,8.86)$ \\
\hline 11 & $(20.03,31.70)$ & $(10,20)$ & $(16.77,36.39)$ & 27 & $(37.40,8.87)$ & $(26,4)$ & $(37.07,10.84)$ \\
\hline 12 & $(17.54,29.11)$ & $(11,19)$ & $(32.52,30.71)$ & 28 & $(39.53,9.00)$ & $(27,3)$ & $(39.06,14.13)$ \\
\hline 13 & $(17.72,31.41)$ & $(12,18)$ & $(19.37,43.07)$ & 29 & $(40.10,7.45)$ & $(28,2)$ & $(47.24,3.83)$ \\
\hline 14 & $(23.09,23.20)$ & $(13,17)$ & $(24.17,26.60)$ & 30 & $(38.88,3.59)$ & $(29,1)$ & $(42.49,2.36)$ \\
\hline 15 & $(26.17,24.25)$ & $(14,16)$ & $(23.24,26.07)$ & 31 & $(43.11,1.32)$ & $(30,0)$ & $(40.17,3.37)$ \\
\hline 16 & $(24.29,24.88)$ & $(15,15)$ & $(21.17,20.70)$ & & & & \\
\hline
\end{tabular}

Fig. 12 shows the positioning errors using different method and their probability distributions (the card reader 1 as the reference point). 


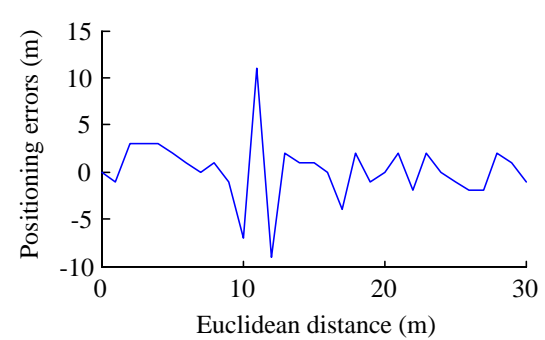

(a) Matching between two kinds of test data

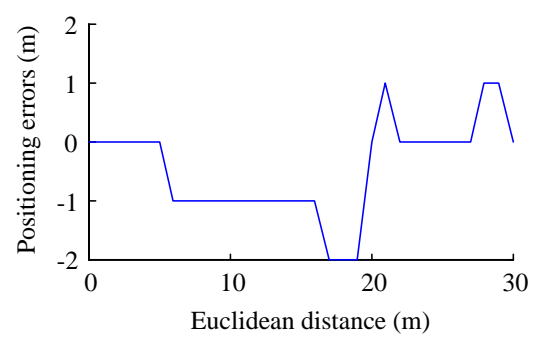

(c) Matching between two kinds of filtering data

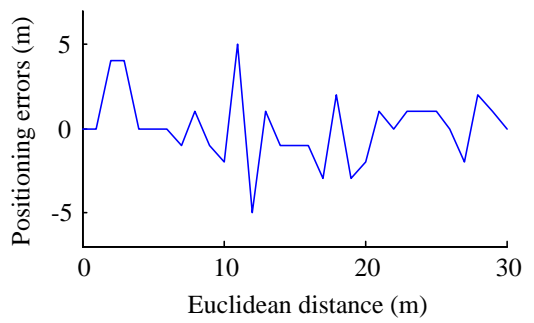

(b) Matching between real-time filtering and database test data

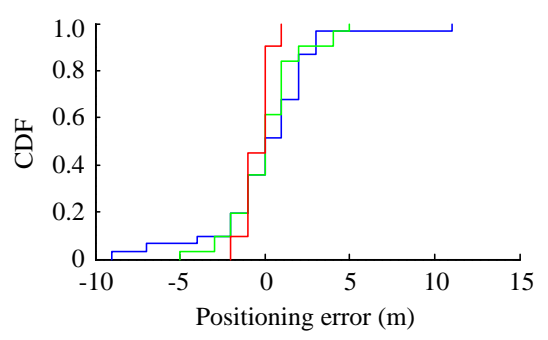

(d) CDF of positioning errors of three methods

Fig. 12. Positioning errors according to Table 1 data using different positioning method

Positioning measurement experiments were performed in tunnels with metallic supports, overhead lines, hydraulic supports and other facilities. Comparing the LFP positioning method directly adopting real-time measured values and database measured values as well as the LFP method of error suppression only for real-time data with the method given in this paper and analyzing them are shown in Fig. 12. From Fig. 12, we can see, the error ranges of the first two methods are respectively from -9 to $11 \mathrm{~m}$ (blue CDF curve of Fig. 12d) and from -5 to $5 \mathrm{~m}$ (green CDF curve of Fig. 12d). When the method given in this paper is adopted, the error range is from -2 to $1 \mathrm{~m}$ (red CDF curve of Fig. 12d) and maximum positioning errors reduce respectively by 9 and $3 \mathrm{~m}$. The positioning result from the measured data is largely in line with the result from the simulation, and therefore the method can provide accurate positioning of personnel in tunnels.

\section{Conclusions}

This paper analyzed the applicability of RSSI and TOA technologies in mine personnel positioning with respect to resolution and suppression over tunnel factors, and described their strength and weakness. Besides, this reseach pointed out the TOA-based positioning was worth of being applied in mine tunnels, and analyzed the problems in applying the TOA-based LFP positioning to position personnel in tunnels and solutions.

This paper established effective algorithms in tunnel environment by the TOA and LFP approach. For the influence of tunnel NLOS time delay error, this paper put forth the de-noising algorithm of database data using the distance threshold limitation and the modified mean filtering (MMF), and the error suppression algorithm of real-time data using speed threshold limitation and MMF. On this basis, the paper developed the nearest neighboring data matching algorithm based on historical location and the speed threshold limitation for personnel LFP positioning.

Simulation and experiment were conducted on the tunnel personnel positioning method based on TOA and modified LFP positioning, and were compared with the basic LFP positioning method and the one using error suppression of the real-time data. The results indicated the positioning error from the method presented in this paper dropped significantly, and the maximal error from the physical experiment declined by 9 and $3 \mathrm{~m}$, respectively. And in the condition used in the experiment the positioning accuracy of $3 \mathrm{~m}$ is achievable.

\section{Acknowledgments}

Project supports from the National Science Foundation of China (No.51134024) and the National High Technology Research and development Program of China (No.2012AA062203) are acknowledged.

\section{References}

[1] Zhai WY, Sun YJ, Xu Z, Li S. Power allocation and mode selection methods for cooperative communication in the rectangular tunnel. International Journal of Mining Science \& Technology,2015;25(02):253-260.

[2] Sun JP, Li CX. In-pit coal mine personnel uniqueness detection technology based on personnel positioning and face recognition. International Journal of Mining Science \& Technology 2013;23(03):357-361.

[3] Hu H, Gao JX, Yao YF. Land deformation monitoring in mining area with PPP-AR. International Journal of Mining Science \& Technology,2014,24(02):207-212.

[4] Fang XQ, Zhao JJ, Hu Y. Tests and error analysis of a self-positioning shearer operating at a manless working face. Mining Science \& Technology 2010;20(01):53-58

[5] Bahl P, Padmanabhan V N. Radar: an in-building rf-based user location and tracking system. In: Proceedings of the Nineteenth Annual Joint Conference of the IEEE Computer and Communications Societies. Piscataway, NJ,USA: IEEE; 2000. p.775-784.

[6] Bisio I, Cerruti M, Lavagetto F, Marchese M, Pastorino M, Pastrorino M, Randazzo A, Sciarrone A. A Trainingless WiFi fingerprint positioning approach over mobile devices. IEEE Antennas \& Wireless Propagation Letters 2014;13:832-835.

[7] Jaegeol Y. Introducing a decision tree-based indoor positioning technology. Expert System with Applications 
2008;34(2):1296-1302.

[8] Heidari M, Pahlavan K. Identification of the absence of direct path in TOA-based indoor localization systems. International Journal of Wireless Information Networks 2008;15(3-4):117-127.

[9] Wang K, Wang QP, Jiang D. A routing and positioning algorithm based on a K-barrier for use in an underground wireless sensor network. Mining Science \& Technology 2011;21(06):773-779.

[10] Liu XW, Zhang XJ, Hao LN, Yu WL, Wang J. Research on underground fingerprint localization algorithm based on WI-FI. Chinese Journal of Sensors \& Actuators 2012;25(06):854-858.

[11] Cui LZ, Li L, Yuan MM, Li Z. Research on underground coal mines positioning algorithms based on kernel function and particle filter. Chinese Journal of Sensors \& Actuators 2013;26(12):1728-1733.

[12] Li L, Ding EJ, Hao LN, Zhang L. An improved location fingerprint localization matching algorithm in coal mine. Chinese Journal of Sensors \& Actuators 2014;27(03):388-393.

[13] Dong JP, Yang C, Lu XL. Underground fingerprint module positioning algorithm based on WiFi. Industry \& Mine Automation 2014;40(10):87-89.

[14] Sun JP, Li CX. Mine TOA positioning method based on Kalman filtering and fingerprint positioning. Journal of China University of Mining \& Technology 2014;43(06):1127-1133.

[15] Sun JP, Li CX. Evaluation method of positioning accuracy for mine underground personnel based on TOA technology. Coal Science \& Technology 2014;42(03):66-68,72.

[16] Emslie A, Lagace R, Strong P. Theory of the propagation of UHF radio waves in coal mine tunnels. IEEE Transactions on Antennas \& Propagation 1975;23(2):192-205.

[17] Sun JP, Cheng LF, Zhang CS. Influence of transverse dimensions on electromagnetic waves propagation in rectangular tunnels. Journal of China University of Mining \& Technology 2005;34(05):596-599.

[18] Moridi MA, Kawamura Y, Sharifzadeh M, Chanda EK, Wagner M, Jang H, Okawa H. Development of underground mine monitoring and communication system integrated ZigBee and GIS. International Journal of Mining Science \& Technology,2015, 25(05):811-818.

[19]Greenstein LJ, Erceg V, Yeh YS, Clark MV. A new path-gain/delay spread propagation model for digital cellular channels. IEEE Trans on Vehicular Technology 1997;46 (2):477-484.

[20] Brunato M, Battiti R. Statistical learning theory for location fingerprinting in wireless LANs. Computer Networks 2005;47(6):825-845.

[21] Fang SH, Lin TN. A dynamic system approach for radio location fingerprinting in wireless local area networks. IEEE Transactions on Communications 2010;58(4):1020-1025.

[22] Narzullaev A, Park Y, Yoo K, Yu J. A fast and accurate calibration algorithm for real-time locating systems based on the received signal strength indication. International Journal of Electronics \& Communications 2011;65(4):305-311. 